Anregungen zur kooperationsfördernden Weiterentwicklung desselben; beides werden Gerichte und Wissenschaft gewiß schon bald wieder gut gebrauchen können.

Herbert Kronke

Günther Jaenicke/Erich Schanze/Wolfgang Hauser

\title{
A Joint Venture Agreement for Seabed Mining
}

Frankfurt, Metzner, 1981, 127 S., DM 35,-

Das Näherrücken der Rentabilitätsschwelle für den Abbau maritimer Rohstoffe hat dem Völkerrecht neue Aufgaben zugewiesen: Die Konflikte zwischen nationalen, universalen, ökonomischen und ökologischen Interessen sind in einer Vielzahl von Seerechtskonferenzen zwar immer neu thematisiert, aber nicht gelöst worden. Die vorliegende Studie untersucht einen Teilbereich der verzweigten Materieden Tiefseebergbau. Dabei beschränkt sie sich auf einen Rohstoff, die Manganknollen, da dessen Besonderheiten bereits technisch und ökonomisch relativ genau erforscht sind und so Interessenkollisionen ziemlich exakt vorhersehbar erscheinen. Die Arbeit entstand aus einem Gutachtenauftrag des Bundesministers für Wirtschaft.

Die Verfasser können auf einen bedeutenden Fundus an Erfahrungen zurückgreifen, welche sie bei der Analyse terrestrischer Rohstoffvorhaben in Entwicklungsländern erworben haben. Diese kommen ihnen insbesondere deshalb zugute, weil ihnen für das vorliegende Projekt kein empirisches Material zur Verfügung steht. Damit dominiert eindeutig die rechtspolitische Perspektive. Anknüpfend an bisherige Vorarbeiten der Seerechtskonferenzen gehen sie von einer gespaltenen Aufgabenverteilung bei der Gewinnung der Manganknollen aus: Die internationale Hohe Behörde übernimmt Verwaltung, Zuteilung und Aufsicht als gemeinsames Organ aller Mitgliedstaaten der Seerechtskonvention; demgegenüber werden Exploration und Abbau der Knollen durch Unternehmen durchgeführt. Hier wird unterschieden zwischen dem Generalunternehmen (enterprise), das in staatlicher oder privater Hand organisiert sein kann. Ihm obliegen die planerischen und organisatorischen Vorarbeiten. Gemeinsam mit Investoren, welche die finanziellen Mittel aufbringen sollen, entsteht ein eigenes Förderunternehmen (operating company), welches die technische Ausführung übernimmt. Sobald die Hohe Behörde einen Generalunternehmer auf dessen Antrag die Lizenz zur Exploration oder zum Abbau in einem bestimmten Gebiet erteilt hat, soll dieses mit den Investoren - und mit Genehmigung der Hohen Behörde- das Förderunternehmen gründen. Auf diesen letzten Vorgang bezieht sich das joint venture agreement, welches in der Untersuchung vorgestellt wird. Es enthält zugleich den Gründungsvertrag des Förderunternehmens und die Genehmigung der Hohen Behörde in der Form des trilateralen Vertrages. So entsteht eine quasi gesellschaftsrechtlich vermittelte Form des Interessenausgleichs, welche sich nach 
den früheren Ergebnissen der Forschungsgruppe bereits bei der terrestrischen Rohstofferschließung bewährt hat.

Nach grundlegenden Einleitungen (S. 9-36), welche die tatsächlichen Vorbedingungen und die von der Arbeitsgruppe jeweils gewählte Lösungsalternative thematisieren, folgt der Text eines Entwurfs zu einem Mangan-Knollen-Abkommen mit knappen Erläuterungen (S. 37-91). Auszüge aus den Zwischenergebnissen der Seerechtskonferenzen (S. 93-119) und eines Auswahlbibliographie (S. 120-127) schließen die Darstellung ab. Der Vertragsentwurf enthält nach einleitenden Bestimmungen über die Lizenzvergabe an den Generalunternehmer und die Projektierung (Teil A) und allgemeinen Regeln über die Rechte und Pflichten der Beteiligten sowie einzelne Leistungsstörungen (Teil B) Vereinbarungen über das Förderunternehmen als Joint Company (Teil C). Dessen Stammkapital soll zu je $50 \%$ vom Generalunternehmer und den Investoren aufgebracht werden (S. 66). Beide Seiten sollen im Bord of Directors mit je 3 Mitgliedern vertreten sein, zu welchen ein neutrales Mitglied hinzutritt (S. 67). Der Board wählt den Generaldirektor (S. 70). Hingegen beschränkt sich die Hohe Behörde auf die unternehmensexterne Aufsicht (S. 55), Beteiligungsrechte kommen ihr nicht zu. Unmittelbar am Förderunternehmen beteiligt sind so lediglich Generalunternehmen und Investoren, also Private, Einzelstaaten oder Staatengruppen, nicht hingegen die internationle Gemeinschaft. Die Verteilung der Gewinne findet ausschließlich zwischen den Beteiligten statt (S. 72), die Hohe Behörde ist durch ertragsorientierte Abgaben beteiligt (S. 105 ff.). Eingehend geregelt ist insbesondere die Berücksichtigung von Entwicklungsländern in allen Stadien der Planung und Durchführung des Bergbaues (S. 48), der Technologietransfern durch Ubertragung von know how (S. 82) und durch Schulung und Ausbildung des Personals (S. 83 f.).

Im Gegensatz zu den vielschichtigen Modellen der Interessenvermittlung im terrestrischen Bergbau weist dieser Vorschlag eine relativ einfache Struktur auf: Der internationalen Gemeinschaft kommt die Verteilung die unternehmensexterne Aufsicht und die Abgabenerhebung zu; Planung, Durchführung und Gewinn stehen den Gesellschaften zu, die überwiegend aus der nördlichen Hemisphäre stammen werden. Ob sich die Mehrzahl der Staaten mit einer solchen Lösung abfinden wird, ist keineswegs sicher. Hier leistet der Vorschlag keine Argumentationshilfe: Warum gerade diese und nicht etwa alternative Modelle gewählt werden, wird nicht dargestellt. Insbesondere bleibt so of fen, warum gerade die hier gezeigten Systeme des Interessenausgleichs anderen überlegen sein sollen. Vielmehr neigen die vielfältigen und durchaus antagonistische Interessenten- und Einflußsphären dazu, hinter mehr technischen Fragen der Gremienpluralität und -zusammensetzung zu verschwinden. Letztere Fragen lassen sich jedoch ohne politische Entscheidung der ersteren kaum lösen. Hier zeigt sich ein grundlegendes Problem des Vorschlages: Inwieweit ist die ausschließliche Beschränkung auf joint-venture-agreements in der Lage, den tatsächlichen Regelungsbedürfnissen gerecht zu werden? Reichen die realen Probleme nicht viel weiter? Woher bezieht die Hohe Behörde ihre Durchsetzungsfähigkeit gegen mächtige staatliche Gesellschaften? Wo sollen die abgebauten Manganknollen verarbeitet werden? Solche und andere Fragen beeinträchtigen nicht den 
Wert der vorliegenden Untersuchung, sondern zeigen vielmehr die Defizite der Erforschung des rechtlichen Umfeldes. Hier kann die Studie nur ein Element darstellen, das ergänzungswürdig, aber auch -bedürftig ist. Sie kann nicht mehr als ein Baustein in einem großen Zusammenhang sein, in welchen sie noch eingeordnet werden muß. Hier ökonomische und politische Bezüge zu verdeutlichen, macht erst den praktischen Wert der rechtlichen Interessenvermittlung aus. Hier waren die früheren Bände der Reihe vorbildlich; an sie sollte bei der weiteren Arbeit angeknüpft werden.

Christoph Gusy

\author{
Karlernst Ringer/Ernst-Albrecht v. Renesse/Christian Uhlig (Hrsg.) \\ Perspektiven der Entwicklungspolitik \\ Horst Erdmann Verlag, Tübingen, 1981, 509 S., DM 68,-
}

Der vorliegende Sammelband faßt nicht nur einzelwissenschaftlich relevante, sondern multi- und interdisziplinär erarbeitete Forschungsergebnisse zusammen (Einführung, S. 13), die sich mit den verschiedensten Gegenständen, Zielen, Strukturen und Methoden auf dem Felde von Entwicklungsvorgängen beschäftigen, aus denen sich eine "Entwicklungspolitik" speist oder speisen kann. Den damit augenfälligen Schwierigkeiten, diese Stoffmasse unter übergeordneten Gesichtspunkten zu gliedern, werden die Herausgeber dadurch Herr, daß sie die Arbeiten in fünf Abschnitten zusammenfassen: Grundlagen und Verläufe, Methodische Ansätze, Strukturelle Aspekte, Strategiefragen und Aktuelle Probleme. Sicherlich hätten die 21 Studien auch anders eingeteilt werden können. Angesichts der Vielzahl von Einzelbeiträgen zu theoretischen Fragen, regelmäßig abgesichert durch Erfahrungen der Verfasser vor Ort, sowie von allgemein gehaltenen Ubersichten (vor allem im 1. Abschnitt) wird nicht recht deutlich, ob überhaupt der Versuch hat gemacht werden sollen, die Thematik unter eine übergeordnete Theoriebildung zu subsumieren. ${ }^{1}$ Der Gewinn in der Auswertung der Einzelbeiträge liegt daher darin begründet, daß sie - Basisdatenkenntnis voraussetzend - den derzeitigen Diskussionsstand zusammenfassend dem interessierten Leser einen präzisen Uberblick verschaffen und von daher deduktiv ausgesuchte Fragen aufwerfen und beantworten können. Der inhaltlichen Vielfalt des Sammelbandes kann in einer Rezension kaum angemessen nachgegangen werden, so daß hier zunächst nur auf die bewußte Ausgrenzung der bildungsöko-

1 Dieser Versuch wird dezidiert im gleichzeitig erschienenen Sammelband Rolf Hanisch/Rainer Tetzlaff (Hrsg.), Staat und Entwicklung - Studien zum Verhältnis von Herrschaft und Gesellschaft in Entwicklungsländern -, Frankfurt a. M.-New York 1981, S. 656, gemacht, indem dort fragend und postulierend dem Begriff und der Funktion des sog. "peripheren Staates" als Analagon zur "peripher-kapitalistischen Gesellschaftsformation" nachgegangen wird. 